\title{
Neuronal correlates of affective theory of mind in schizophrenia out-patients: evidence for a baseline deficit
}

\author{
D. Mier ${ }^{1,2 *}$, C. Sauer ${ }^{1}$, S. Lis ${ }^{3}$, C. Esslinger ${ }^{1}$, J. Wilhelm ${ }^{3}$, B. Gallhofer ${ }^{3}$ and P. Kirsch ${ }^{1,4}$ \\ ${ }^{1}$ Division for Imaging in Psychiatry, Central Institute of Mental Health, Mannheim, Germany \\ ${ }^{2}$ Department for Clinical Psychology, Central Institute of Mental Health, Mannheim, Germany \\ ${ }^{3}$ Centre for Psychiatry and Psychotherapy, University of Giessen, Germany \\ ${ }^{4}$ Mannheim School of Medicine, University of Heidelberg, Germany
}

Background. Schizophrenia out-patients have deficits in affective theory of mind (ToM) but also on more basal levels of social cognition, such as the processing of neutral and emotional expressions. These deficits are associated with changes in brain activation in the amygdala and the superior temporal sulcus (STS). However, until now there have been no studies that examined these different levels of social cognition and their neurobiological underpinnings in patients within one design.

Method. Sixteen medicated schizophrenia out-patients and 16 matched healthy controls were studied with functional magnetic resonance imaging (fMRI) during a social cognition task that allows the investigation of affective ToM (aToM), emotion recognition and the processing of neutral facial expressions.

Results. Patients showed a deficit in emotion recognition and a more prominent deficit in aToM. The performance in aToM and in emotion recognition was correlated in the control group but not in the schizophrenia group. Region-ofinterest analysis of functional brain imaging data revealed no difference between groups during aToM, but a hyperactivation in the schizophrenia group in the left amygdala and right STS during emotion recognition and the processing of neutral facial expressions.

Conclusions. The results indicate that schizophrenia out-patients have deficits at several levels of social cognition and provide the first evidence that deficits on higher-order social cognitive processes in schizophrenia may be traced back to an aberrant processing of faces per se.

Received 4 December 2008; Revised 13 November 2009; Accepted 16 November 2009; First published online 8 January 2010

Key words: Emotion recognition, fMRI, schizophrenia, social cognition, theory of mind.

\section{Introduction}

Schizophrenia is a psychiatric disorder associated with deficits in different processes of social cognition on the behavioural and also on the neuronal level (Pinkham et al. 2003; Penn et al. 2008). Social cognitions comprise all information processes that culminate in the accurate perception of the dispositions and intentions of others, such as the recognition of identity, movement and facial expressions (Brothers, 1990). Theory of mind (ToM) is the ability to infer mental states to others, such as wishes, desires and intentions (Premack \& Woodruff, 1978). Thus, ToM is a level

\footnotetext{
* Address for correspondence: D. Mier, Division for Imaging in Psychiatry, Central Institute of Mental Health, J5, D-68159 Mannheim, Germany.

(Email : Daniela.Mier@zi-mannheim.de)
}

of social cognition that is preceded by several information processing steps.

In the case of schizophrenia, disturbances in ToM have been found repeatedly (Corcoran et al. 1997; Doody et al. 1998; Kington et al. 2000). However, an important differentiation in the assessment of ToM is whether 'cold' or 'hot' aspects of ToM are investigated. 'Cold' aspects relate to cognitive aspects and 'hot' aspects to affective aspects (Brothers \& Ring, 1992). Affective ToM (aToM) is most often investigated by the understanding of faux pas or irony (e.g. Shamay-Tsoory et al. 2005), or with the eyes test, where a mental state is inferred by the expression of the eyes region (Baron-Cohen et al. 1999). Cognitive ToM is most often tested by the ability to infer a mental state from a person (first-order ToM) or to infer what a person is thinking about the mental state of a third person (second-order ToM; e.g. Gregory et al. 2002). Most paradigms for cognitive ToM exclude emotional 
information (e.g. Brüne, 2003; Brunet et al. 2003) and it has been shown that schizophrenia out-patients have a deficit in cognitive ToM (Janssen et al. 2003; Greig et al. 2004; Kelemen et al. 2005; Inoue et al. 2006; Horan et al. 2009). Of note, the studies that investigated both affective and cognitive ToM in schizophrenia out-patients found more evidence for a specific deficit in aToM than for a global ToM deficit (Herold et al. 2002; Shamay-Tsoory et al. 2007; Mo et al. 2008; Kern et al. 2009).

However, in schizophrenia deficits were found not only in ToM but also on more basal levels of social cognitions, as in the processing of neutral faces (Manor et al. 1999) and in emotion recognition (ER; for reviews see Mandal et al. 1998; Edwards et al. 2002). These deficits in the processing of neutral faces and in ER were also shown to occur in schizophrenia outpatients (Addington \& Addington, 1998; Penn et al. 2000; Loughland et al. 2002; Johnston et al. 2006). Taken together, the results from these behavioural studies indicate that schizophrenia out-patients have deficits on at least three levels of social cognition: the processing of neutral faces, ER and aToM.

Brothers (1990) found that the amygdala, the superior temporal sulcus (STS) and the medial orbitofrontal cortex act as the neuronal network for social cognition. Functional imaging studies investigating social cognition in schizophrenia found alterations in two areas of the social cognition network predominantly, namely the amygdala and the STS. Studies investigating the passive viewing of neutral faces found hyperactivity in the amygdala and hippocampus (Holt et al. 2006; Surguladze et al. 2006) and the STS (López-lbor et al. 2008) in schizophrenia patients. By contrast, the results for the processing of emotional faces are more heterogeneous. Most studies found amygdala hypoactivation in schizophrenia (Gur et al. 2002; Hempel et al. 2003; Fakra et al. 2008; see Li et al. 2009 for a meta-analysis) whereas others found hyperactivation in patients (Kosaka et al. 2002; Holt et al. 2006). Functional imaging studies investigating ToM in schizophrenia showed no difference in the amygdala activation and mixed results for STS activation, but all studies found prefrontal hypoactivation (Russell et al. 2000; Brunet et al. 2003; Andreasen et al. 2008; Brüne et al. 2008). These results from functional imaging studies together indicate that a pattern of hyperactivation in areas of the social cognition network occurs during the passive viewing of neutral facial stimuli. ER tasks lead to heterogeneous results in areas of the social cognition network and, if the task gets more complex, as in ToM tasks, hypoactivation in prefrontal areas also occurs.

The question that arises is whether these deficits occur independently on each of these levels of social cognition or whether deficits on a higher level of social cognition (aToM) can be traced back to deficits on lower levels of social cognitions, such as the processing of faces itself or ER. However, although these behavioural and brain functional deficits on different levels of social cognition in schizophrenia are known, to our knowledge no functional imaging study has investigated the neuronal correlates within one paradigm. Thus, the aim of the present study was to investigate the neuronal correlates of aToM, ER and processing of faces with neutral expressions in schizophrenia out-patients with a modified version of a new aToM task. Using this task in a healthy control population, we found a close relationship between ER and ToM in the behavioural data and in terms of neuronal activation and we have shown that the task is suited to elicit activation in the amygdala and the STS (Mier et al., unpublished data). We hypothesized that schizophrenia out-patients show a deficit in aToM, ER and processing of neutral faces. Furthermore, we suggested that, on a neuronal level, this deficit in aToM can be traced back to changes in brain activation in the amygdala and the STS during ER and the processing of neutral facial expressions.

\section{Method}

Sample

Sixteen schizophrenia out-patients and 16 healthy controls participated in the study. The groups were matched for gender, age and education, and also fluid and crystallized intelligence (Table 1). Almost all participants were right-handed; only one schizophrenia out-patient was left-handed. No participant had a history of neurological disease. To exclude actual or life-time psychiatric diagnoses, healthy controls were screened by means of the Mini-DIPS (Diagnostisches Kurz-Interview bei psychischen Störungen; Margraf, 1994). Controls with first relatives with any history of psychiatric disorders were excluded. All patients met criteria for DSM-IV diagnosis of schizophrenia and were on stable doses of antipsychotic medication. Some of them were receiving additional medication (Table 1). Psychopathology of the patients was measured using the Scale for the Assessment of Positive Symptoms (SAPS; Andreasen, 1984) and the Scale for the Assessment of Negative Symptoms (SANS; Andreasen, 1983). Schizophrenia out-patients showed only moderate symptom severity (Table 1). Participants gave their written informed consent prior to participating in the study. The study was approved by the local ethics board of the University of Giessen School of Medicine. 
Table 1. Sociodemographic variables for both groups

\begin{tabular}{llll}
\hline & $\begin{array}{l}\text { Schizophrenia } \\
\text { out-patients }\end{array}$ & $\begin{array}{l}\text { Healthy } \\
\text { controls }\end{array}$ & $\begin{array}{l}\text { Significance } \\
p \text { value }\end{array}$ \\
\hline Gender, male/female & $11 / 5$ & $11 / 5$ & $1.0, \chi$, N.S. \\
Age (years), mean (s.D.) & $34.25(6.95)$ & $37.0(8.18)$ & 0.31, N.S. \\
Education (years), mean (s.D.) & $11.44(1.63)$ & $10.88(1.86)$ & 0.54, N.S. \\
Crystallized intelligence, mean (s.D.) & $110.06(14.29)$ & $113.38(14.08)$ & 0.51, N.s. \\
Fluid intelligence, mean (s.D.) & $114.31(15.86)$ & $118.06(18.97)$ & 0.55, N.S. \\
SAPS score & $1.5(1.37)$ & & \\
SANS score & $7.94(2.86)$ & & \\
Chlorpromazine equivalent & $901.59(647.1)$ & & \\
\hline
\end{tabular}

SAPS, Scale for the Assessment of Positive Symptoms; SANS, Scale for the Assessment of Negative Symptoms; S.D., standard deviation; N.S., not significant.

\section{Procedure}

Data were acquired at two sessions within 1 week. The interviews and the intelligence measures were conducted in the first session and the functional magnetic resonance imaging (fMRI) study in the second. Crystallized intelligence was measured by means of the multiple-choice vocabulary test MWT-B (Mehrfach Wortschatz-Test Form B; Lehrl, 1977) and fluid intelligence was assessed by means of the KAI (Kurztest für Allgemeine Intelligenz; Gallwitz et al. 1992). Before the aToM task, an adaptive ER task was performed, using the same stimuli (data not presented here). Stimuli consisted of morphed photographs of four males and four females with facial expressions of joy, anger, fear and a neutral expression. The applied stimuli had been rated previously by 160 undergraduate students and only emotional expressions with a recognition rate between $70 \%$ and $90 \%$ were included.

Each trial started with the display of a statement, followed by a face stimulus. Three different conditions were implemented by the statements: aToM, ER and neutral facial processing. Stimuli in the aToM and the ER condition were identical. The stimuli in the neutral condition consisted of the same persons, but only stimuli with neutral facial expressions were used. Subjects had to judge whether the depicted statement matched the picture of the person. In the aToM condition the task was to judge whether the shown person was going to carry out a certain action. In the ER condition subjects had to recognize the emotion. In the neutral condition the task was to decide whether a particular physical feature was present in the depicted person. For both the aToM and the ER condition, to solve the task the emotional content had to be explored. Responses were given by a button press on a response panel with index and middle finger for 'yes' and 'no' answers respectively (see Fig. 1 for experimental design).

The statement was displayed for $2 \mathrm{~s}$, followed by the picture, which was displayed for a maximum of $2 \mathrm{~s}$. If the response was faster than $2 \mathrm{~s}$, the picture was terminated and a fixation cross was depicted for the rest of the trial. During the inter-trial interval (ITI) the fixation cross was also shown. The mean ITI was $2 \mathrm{~s}$ (jittered between 0.5 and $3.5 \mathrm{~s}$, resulting in a stimulus onset asynchrony of $4.5-7.5 \mathrm{~s}$ ). Each condition was presented 30 times. The conditions and the categories within the conditions and the stimulus subjects were presented in a pseudo-randomized order (no more than two repetitions in succession). The total duration of the experiment was around $9.5 \mathrm{~min}$.

\section{Data acquisition and data analysis}

Functional MRI data were collected with a 1.5-T General Electrics (USA) whole-body scanner; 185 volumes were collected with an axial $\mathrm{T} 2 *$-weighted echo planar sequence [ 30 slices, slice thickness $5 \mathrm{~mm}$, repetition time (TR) $3000 \mathrm{~ms}$, echo time (TE) $50 \mathrm{~ms}$, alpha $90^{\circ}$, field of view (FoV) $220 \mathrm{~mm}, 64 \times 64$ matrix]. Slices were adjusted to AC-PC.

Behavioural data were analysed by means of SPSS version 13 (SPSS Inc., USA). Reaction time and performance data were analysed with $2 \times 3$ factorial ANOVAs, with the between-subject factor group and the repeated-measures factor task condition. Subsequent two-sample $t$ tests were used to identify differences between groups within each condition and dependent $t$ tests to assess differences between the conditions within the whole sample. Relationships between performance data in the three conditions 


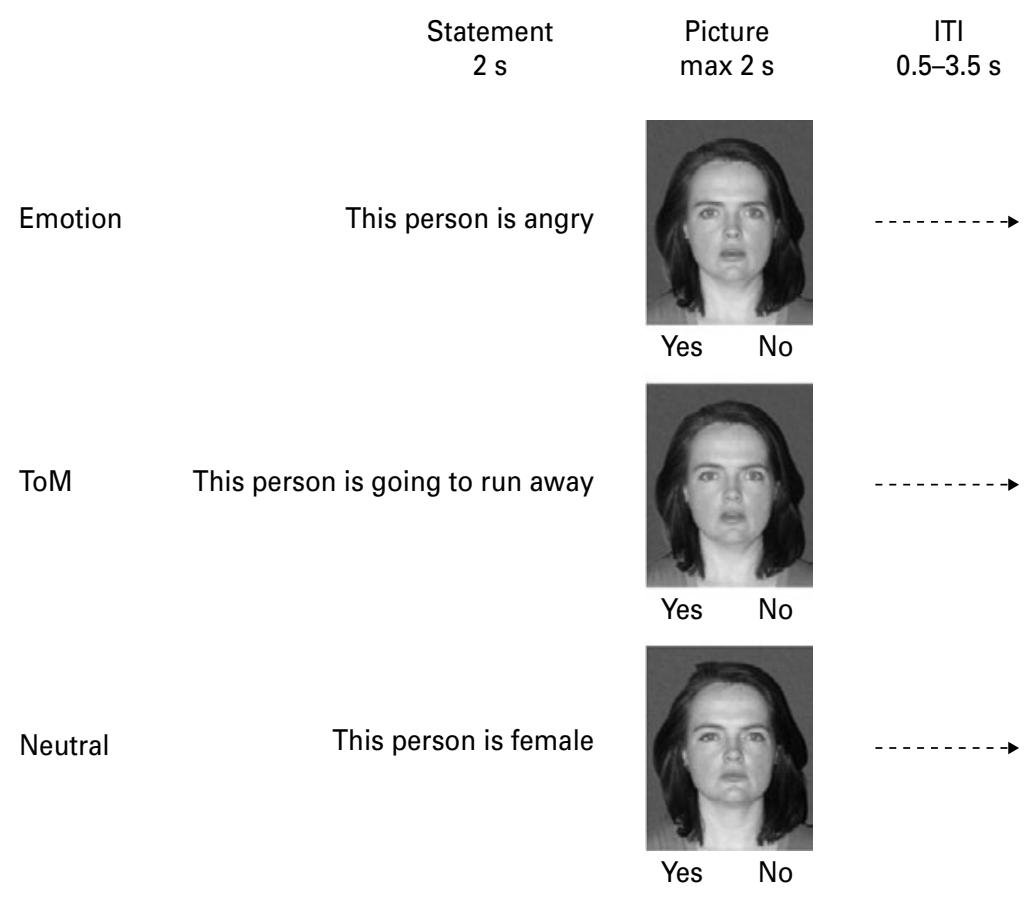

Fig. 1. Experimental conditions and procedure shown for a single trial for each condition: emotion recognition (Emotion), affective theory of mind (ToM) and neutral facial processing (Neutral).

were analysed by calculating Pearson productmoment correlations.

Functional imaging data were preprocessed and analysed using SPM2 (www.fil.ion.ucl.ac.uk/spm/ software/spm2/). Preprocessing consisted of realignment, slice time correction, normalization and smoothing [ $8 \mathrm{~mm}$ full-width at half-maximum (FWHM)]. A study-specific template was used for normalization.

For the statistical analysis, a fixed-effects general linear model was calculated for each person separately. The onsets of all trials with correct responses were used as predictors separately for each condition. Furthermore, one regressor was defined with all trials with wrong responses across the three conditions. Regressors were convolved with a haemodynamic response function. Realignment parameters (translations and rotations) were used as covariates. A 128-s high-pass filter was applied. After model estimation, linear contrasts were applied directly comparing all conditions and testing each condition against baseline. Those individual linear contrasts were used in a second-level mixed-effects model. All comparisons were conducted with $t$ tests. For group comparisons, region of interest (ROI) analyses were conducted for the amygdala and the STS using the small volume correction as implemented in SPM2. To test for influences of task difficulty and medication on brain activation in the patient group, correlations between those measures and brain activation within each ROI were calculated. To test for associations between altered brain activation in schizophrenia patients and deficits on higher levels of social cognition, correlations between activation during neutral face processing and ER, and behavioural performance during aToM, and vice versa, were conducted in those ROIs, where group differences occurred.

Masks for the ROI analyses were created with MARINA (Walter et al. 2003). The mask for the amygdala was taken from the Anatomical Automatic Labelling (AAL) atlas (Tzourio-Mazoyer et al. 2002). The mask for the STS was taken from the functional imaging result of the comparison of ToM and ER in 40 undergraduate students in the study by Mier et al. (unpublished data).

Because SPM2 does not allow factorial designs to be conducted, we used SPM5 to apply a full factorial model to the data to test for interaction effects between group and condition.

The results of the whole-brain analyses for each group are reported at a significance level of $p<0.05$ false discovery rate-corrected, and for the group comparisons with $p<0.001$ uncorrected, mean Cluster size 5 voxels (see Supplementary Tables S1-S3, available online). The results of the factorial analysis are reported by a slightly more lenient threshold $(p<$ 0.002). From the ROIs, all voxels with a significance level of $p<0.005$ were incorporated into the small volume correction analysis but from all suprathreshold clusters only those with a peak voxel significantly 
activated at $p<0.05$ family-wise error (FWE) corrected are reported.

\section{Results}

\section{Behavioural data}

The MANOVA of the percentage of correct answerers revealed a significant main effect for condition $[F(2,29)=28.229, p<0.001]$. Post-hoc $t$ tests revealed significant differences between all of the conditions $(p<0.05)$. The neutral task was found to be the most difficult and the ER task the easiest. Regarding group effects, the MANOVA of the percentage of correct answers revealed a trend towards significance for the main effect of group $[F(1,30)=3.4, p=0.075]$ and also for the group by condition interaction $[F(2,29)=2.43$, $p=0.096$ ]. Post-hoc comparisons revealed a significantly impaired performance of the patients in the aToM task $[T(30)=2.17, p=0.038]$, a trend of worse performance in the ER task $[T(30)=1.96, p=0.060]$ and no difference in the performance in the neutral task. The MANOVA of reaction times also revealed a significant main effect for condition $[F(2,29)=21.885$, $p<0.001$ ], with longer reaction times during aToM and ER conditions compared to the neutral condition $(p<0.001)$. By contrast, the ToM and the ER conditions did not differ significantly. For reaction times, no significant main effect for group but a significant group by condition interaction $[F(2,29)=3.86, p=0.033]$ was found. Post-hoc $t$ tests revealed a significant reaction time difference between groups for the ER task $[T(29)=2.207, p=0.035]$ but not for either the aToM or the neutral task. The results for the MANOVAs are displayed in Fig. 2.

Correlation analysis for the percentage of correct responses revealed a significant correlation between performance in the aToM and the ER tasks for the healthy controls $(r=0.736, p<0.001)$. By contrast, for the schizophrenia patients a significant correlation was only observed between performance in the ER and the neutral conditions $(r=0.599, p=0.014)$.

\section{Functional brain imaging data}

Whole-brain comparisons between groups revealed significant differences only at an uncorrected significance level $(p<0.001)$ for all three tasks (see Supplementary Tables S1-S4, available online). For the neutral task ROI analysis revealed higher activation in the patients compared to the healthy controls in the left amygdala (peak voxel: $-15,-3,-15 ; T=$ 3.33, $p_{\mathrm{FWE}}=0.037, k=12$; Fig. $3 a$, upper panel) and the right STS (peak voxel: 51, $-60,12 ; T=3.07$, $p_{\mathrm{FWE}}=0.051, k=12$; Fig. $3 a$, lower panel). For ROIs, no (a)

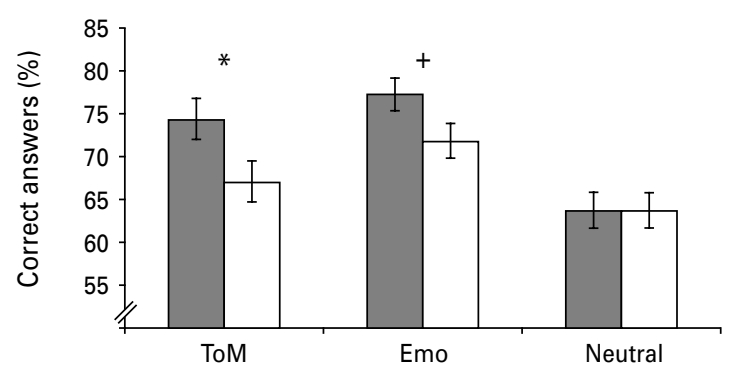

(b)

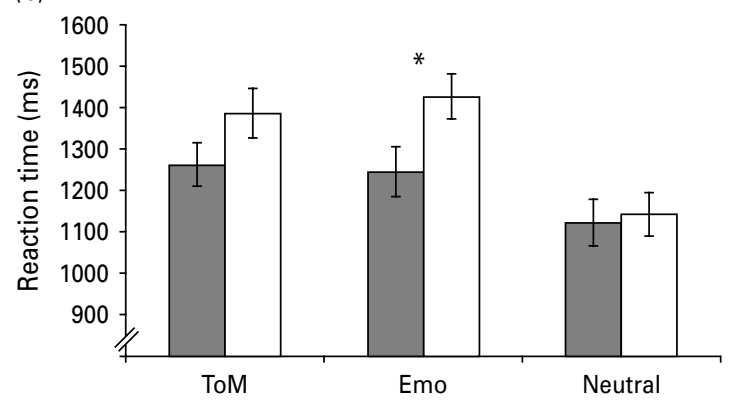

Fig. 2. Behavioural results: mean ( \pm standard error) percentage of $(a)$ correct answers and $(b)$ reaction times for the three experimental conditions and for both groups. $\square$, Schizophrenia patients; $\square$, healthy controls; ToM, affective theory of mind; Emo, emotion recognition; Neutral, neutral facial processing. ${ }^{*} p<0.05,{ }^{+} p<0.1$.

stronger activation was found for controls compared to patients.

ROI analysis of the ER activation again revealed hyperactivation in the left amygdala (peak voxel: $-33,-3,-18 ; T=3.52, p_{\mathrm{FWE}}=0.028, k=17$; Fig. $3 b$, upper panel) and right STS (peak voxel: 51, $-57,12$; $T=5.18, p_{\mathrm{FWE}}=0.001, k=41$; Fig. $3 b$, lower panel). Neither whole-brain analysis nor ROI analysis revealed regions with stronger activation in the controls compared to the patients.

ROI analysis revealed no group differences for the aToM task, neither for the amygdala nor the STS.

Because the visual inspection of the results from the ROI analyses implied a different amount of hyperactivation in patients for the different tasks (see Fig. 4), we tested for interaction effects between group and task. We found a significant group by task interaction for the right STS (peak voxel: $48,-42,18 ; k=15$, $\left.T=3.45, p_{\text {unc }}<0.001\right)$, reflecting a differential relationship between activation between the groups for the ER and aToM tasks. This interaction was based on the fact that healthy controls showed an increase in activation from ER to aToM and schizophrenia patients a decrease (see Supplementary Table S4). For the left amygdala we also found a significant interaction (peak voxel: $-24,-12,-12 ; k=5, T=3.09, p_{\text {unc }}=0.001$ ). 

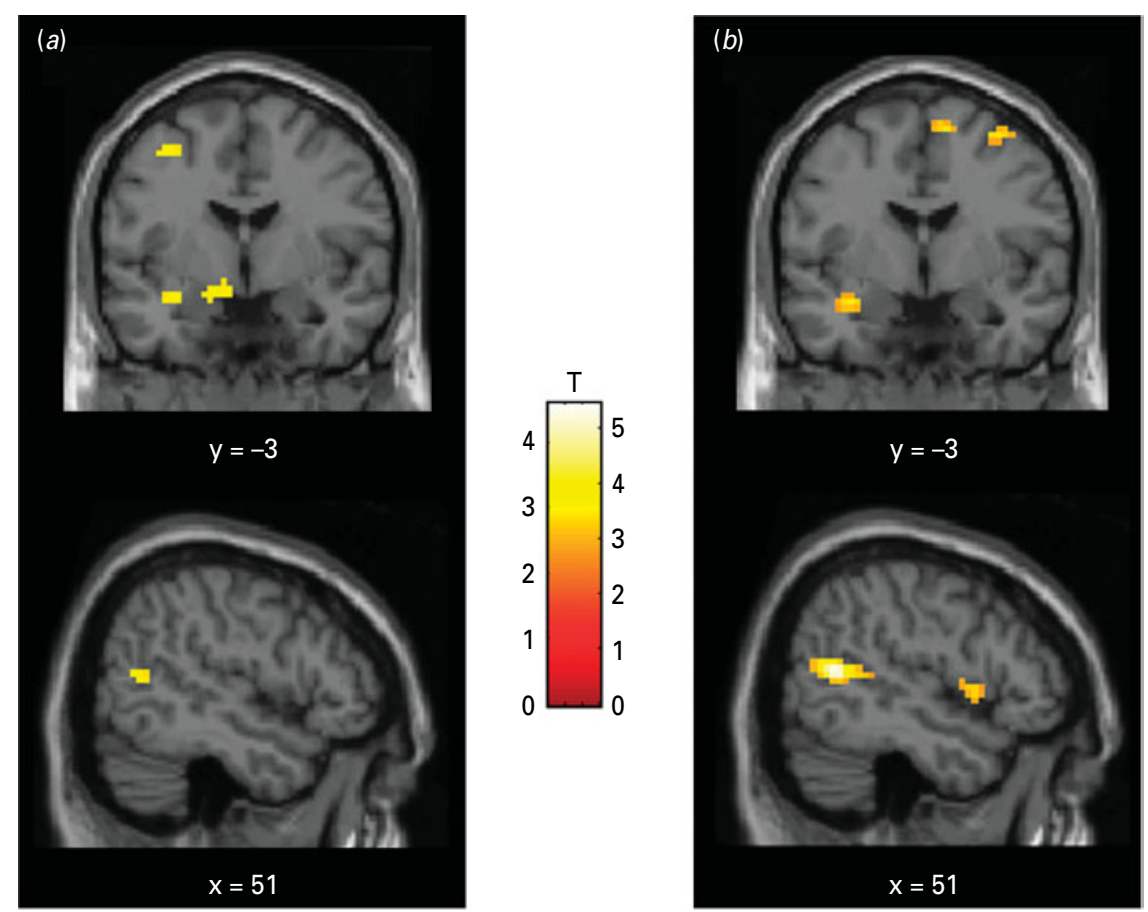

Fig. 3. Significantly increased activation in schizophrenia patients compared to healthy controls in the left amygdala and the right superior temporal sulcus. (a) Neutral condition. (b) Emotion recognition. Threshold for display: $p<0.005$ uncorrected.

However, the difference between the aToM and the neutral condition was not significant $\left(p_{\text {unc }}<0.001\right)$ either in the control group or in the patient group. Even with our liberal threshold no other voxels showed a significant group by task interaction.

Correlation analyses between behavioural results and brain activation revealed a significant association between percentage of correct answers and brain activation in the right STS (peak voxel: 51, $-51,18$; $k=8, T=3.91, p_{\mathrm{FWE}}=0.035$ ) and left amygdala (peak voxel: $\left.-24,6,-27 ; k=9, T=5.54, p_{\mathrm{FWE}}=0.005\right)$ in the control group during ER. In addition, percentage of correct answers during ER was at a trend level correlated to brain activation in the right STS during aToM (peak voxel: $45,-57,18 ; k=8, T=3.5, p_{\mathrm{FWE}}=0.063$ ). No other significant correlations between brain activation and both performance and medication were found within the ROIs using the small volume correction method.

\section{Discussion}

This study was conducted to investigate the neuronal correlates of three different social cognition processes in schizophrenia out-patients: aToM, ER and processing of neutral faces. It was hypothesized that schizophrenia patients have deficits in aToM, ER and the processing of faces itself and that the deficits in aToM can be traced back to activation changes in the amygdala and STS during ER and processing of neutral faces.

On a behavioural level, patients showed deficits in both ER and aToM. In comparison to the control subjects, patients recognized the emotions slower but showed only a trend towards a reduced number of correctly recognized emotions. During the aToM condition, patients recognized fewer intentions correctly but showed no difference in reaction time in comparison to the healthy controls. An explanation for this pattern of behavioural differences could be that schizophrenia patients changed strategy, based on a profound deficit in aToM, from a slower and more accurate processing during the ER task to a less accurate but faster response during the more difficult ToM task. The correlation analyses of performance data in terms of the number of correct answers are in line with this interpretation. While healthy controls showed a significant correlation between the performance during ER and aToM, schizophrenia patients showed a significant correlation between the performance during the neutral condition and the ER condition. This result replicates the finding of Brüne (2005), who found a correlation between ToM and ER in healthy controls but not in schizophrenia 
(a)

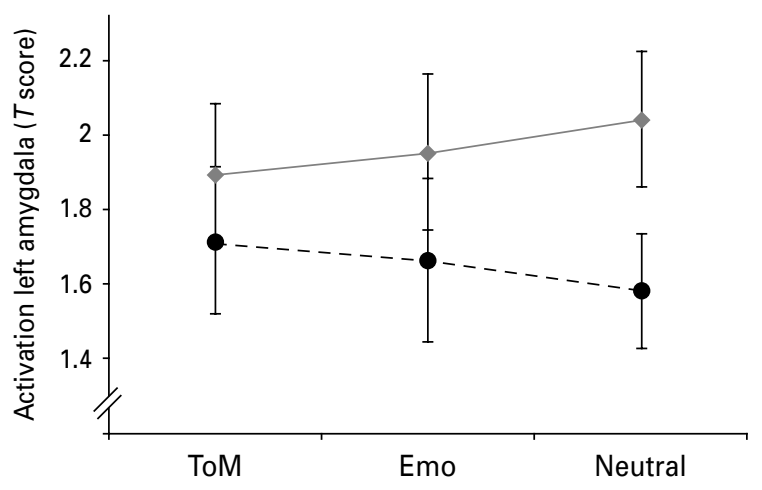

(b)

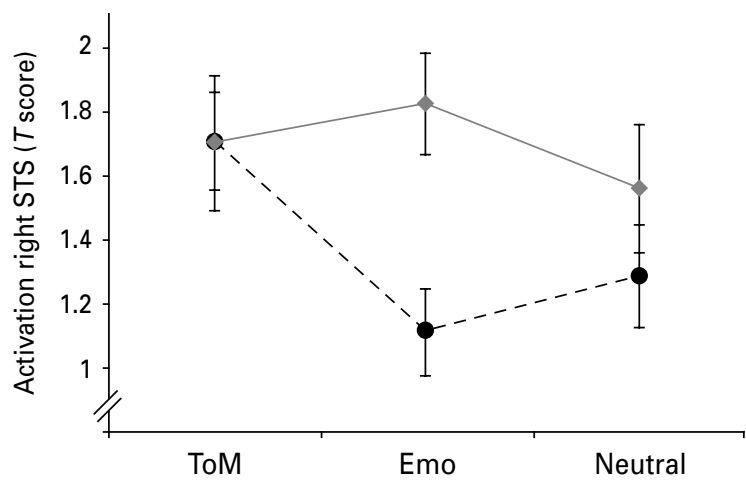

Fig. 4. Means of the maximal $T$ scores for each participant within a region of interest (ROI) for the three experimental conditions and for both groups. (a) Peak activation in the left amygdala. (b) Peak activation in the right superior temporal

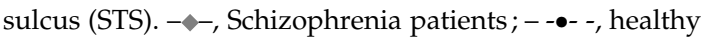
controls; ToM, affective theory of mind; Emo, emotion recognition; Neutral, neutral facial processing.

in-patients. The ability to recognize emotions and mental states seems to be related in healthy controls, but is dissociated in schizophrenia patients. This dissociation between performance in ER and ToM in schizophrenia patients might be based on a change of strategy, caused by severe deficits in ToM. Thus, on a behavioural level, schizophrenia out-patients show deficits in ER and aToM, but the deficit in aToM is more pronounced.

Analysis of the fMRI data revealed significant interactions between group and condition. Patients showed hyperactivation in the left amygdala and right STS during ER and the neutral facial expressions task. However, during aToM there was no difference in these two areas between groups. As in our study, in a study by Pinkham et al. (2008) on trustworthiness, a process associated with ToM, patients without acute paranoid symptoms also showed no differences in the amygdala and STS in comparison to a healthy control group. However, when analysing the age control task,
Pinkham et al. (2008) found hyperactivation in the schizophrenia group compared to the control group in both the amygdala and STS.

Schizophrenia patients seem to have hyperactivation in brain areas of the social cognition network on lower levels of social cognition, but not on higher levels as in ToM. This conclusion is in line with the ToM studies that found hypoactivation in frontal areas in schizophrenia, but no hyperactivation in areas of the social cognition network (Russell et al. 2000; Brunet et al. 2003), and with studies that showed hyperactivation in the amygdala and STS during the viewing of neutral facial expressions (Holt et al. 2006; Surguladze et al. 2006; López-Ibor et al. 2008). An explanation for this phenomenon might come from the working memory literature. Callicott et al. (2003) described activation in the prefrontal cortex as an inverted U-shaped curve, dependent on task difficulty. This U-shaped relationship might also be valid for activation in the amygdala and STS. In the current study, as in our recent study (Mier et al., unpublished data), analysis of behavioural data showed that the aToM task was more difficult than the ER task. In our recent study (Mier et al., unpublished data), the comparison of aToM and ER revealed higher activation for aToM in the STS and in the left amygdala. In the current study whole-brain analysis revealed higher activation in the STS in healthy controls, bilaterally during aToM compared with during ER (see Supplementary Table S4 for details). This difference was not significant in the schizophrenia out-patients. By contrast, the patients' activation within the right STS was stronger during ER than during ToM (Supplementary Table S4). Fig. 4 also reveals a significant activation decrease in the right STS and a slight but non-significant activation decrease in the left amygdala in the schizophrenia patients from ER to aToM but an increase in activation in both structures in the healthy controls. In addition, in the healthy controls, but not in the schizophrenia patients, the performance during ER was related to the activation in the right STS during aToM. This is in line with the dissociation of ER and aToM performance in the schizophrenia out-patients. Thus, based on the hyperactivation during the processing of faces per se, schizophrenia out-patients might have 'a higher start point' for the three social cognition processes than the healthy controls and might have already reached a plateau in brain activation during ER. Whereas the healthy controls are on the ascending side of the inverted U-curve, ER seems to have the optimal difficulty for schizophrenia patients, hindering a further activation increase from ER to aToM. Of note, this interaction effect between group and condition can be traced back to different tasks for the 
left amygdala and right STS (see Fig. 4). Whereas for the amygdala, the largest difference between groups had already occurred during the neutral task completion, for the STS the largest group differences occurred for the ER. This result might reflect a ceiling effect in the amygdala activation in the neutral condition whereas the patients can further increase their STS activation, potentially compensating for deficits in emotion processing. As a result, in schizophrenia more demanding tasks go along with greater behavioural deficits in social cognition but less activation differences in the amygdala and STS. Taken together, the results point to a deficit in early processing steps of social cognitions in schizophrenia and a dissociation of aToM performance from these earlier social cognition processes. However, because we could not find a correlation between activation during the processing of neutral facial expressions and aToM performance, our hypotheses of the aToM deficit in schizophrenia as based on aberrant processing in more basal processes could not be confirmed directly. On a neurophysiological level, there is stronger evidence from our study for an important role of the right STS in social cognition deficits of schizophrenia patients than for an important role of the left amygdala. Thus, the dependence of deficits in higher-order social cognitions, such as aToM, on aberrant brain activation in the social cognition network during lower-order processes, such as face processing per se, should be investigated further in future studies with larger sample sizes, allowing more reliable conclusions from correlation analyses.

Hyperactivation during the processing of neutral facial expressions in schizophrenia may also explain the hypoactivation in the amygdala and STS often found in other studies. In most papers difference contrasts are reported; for example, recognition of emotions versus age judgements. The results of our study, like those of other studies analysing the neutral condition separately (e.g. Pinkham et al. 2008; Seiferth et al. 2008), indicate that the hypoactivation found may be traced back to a hyperactivation during the baseline condition. Therefore, a non-facial baseline condition or the use of an event-related design might be a more appropriate approach to investigate the response to emotional faces in schizophrenia patients.

A possible consequence of this hyperactivation in the left amygdala and the right STS in schizophrenia during the processing of faces with neutral expressions and during ER is an augmented attribution of intentions to others. This assumption is in line with the proposed role of the amygdala in fear conditioning (LeDoux, 2007), the attribution of salience in the extended amygdala (Liberzon et al. 2003) and the construction of self-reference (Grèzes et al. 2006) and the role of the STS in the attribution of intentions (Castelli et al. 2000; Pelphrey et al. 2004; Saxe et al. 2004). The results of this study indicate, on the one hand, that schizophrenia patients need a higher compensatory brain activation to show the same performance rate in the processing of faces with neutral expressions and in ER as healthy controls. On the other hand, this hyperactivation may contribute to hypermentalizing, which is thought to lead to paranoid symptoms and delusions in schizophrenia (Frith, 1992).

Factors that might have influenced brain activation in the patient group could have been medication, task difficulty and positive and negative pathology. All patients received antipsychotic medications, but antipsychotics are not well suited to influencing the processing of emotional information in the amygdala and to improving ER (Pinkham et al. 2007). In addition, the salience reducing effect of antipsychotic medication (Mizrah et al. 2005) would predict hypo- rather then hyperactivation. Moreover, correlation analyses confirmed that the hyperactivation found in patients cannot be explained by medication or performance effects. The hyperactivation in the left amygdala and right STS can be seen as risk factors for positive symptoms, but it is unlikely that they were caused by positive pathology in this study because the patients were more or less free of positive symptoms. As patients in this study showed fairly high levels of negative symptoms, these might have influenced the results. However, negative symptoms tend to be associated with a lack of amygdala activation in emotional processing (Fahim et al. 2005) rather than with amygdala hyperactivation. Overall, hyperactivation in the left amygdala and right STS during the processing of neutral facial expressions and during ER seems to be a trait marker for schizophrenia rather than caused by medication, task performance or pathology.

One puzzling result in the behavioural data is the low performance in the neutral task. Post-hoc analysis revealed that this was due to one poor item in this condition; most subjects did not know the meaning of one word used ('brunette'), leading to many incorrect 'no' answers. However, because this happened equally in both groups, it should not influence the results presented here.

In conclusion, we found deficits in schizophrenia out-patients in ER and aToM and hyperactivation in the left amygdala and right STS during the processing of neutral facial expressions and during ER, but no changes in brain activation during aToM. In schizophrenia patients the ability to recognize affective intentions was dissociated from the ability to recognize emotions. These results provide some evidence that deficits in higher-order social cognitions in 
schizophrenia can be traced back to an aberrant processing of faces per se. However, this possibility should be addressed further in future studies.

\section{Note}

Supplementary material accompanies this paper on the Journal's website (http:/ /journals.cambridge.org/ psm).

\section{Acknowledgements}

D.M. was supported by a fellowship for doctoral students from the University of Giessen. We thank K. Zygrodnik for help with data collection and special thanks go to all the participants.

\section{Declaration of Interest}

None.

\section{References}

Addington J, Addington D (1998). Facial affect recognition and information processing in schizophrenia and bipolar disorder. Schizophrenia Research 32, 171-181.

Andreasen NC (1983). The Scale for the Assessment of Negative Symptoms (SANS). The University of Iowa: Iowa City.

Andreasen NC (1984). The Scale for the Assessment of Positive Symptoms (SAPS). The University of Iowa: Iowa City.

Andreasen NC, Calage CA, O'Leary DS (2008). Theory of mind and schizophrenia: a positron emission tomography study of medication-free patients. Schizophrenia Bulletin 34, 708-719.

Baron-Cohen S, Ring HA, Wheelwright S, Bullmore ET, Brammer MJ, Simmons A, Williams SCR (1999). Social intelligence in the normal and autistic brain. European Journal of Neuroscience 11, 1891-1898.

Brothers L (1990). The social brain: a project for integrating primate behavior and neurophysiology in a new domain. Concepts in Neuroscience 1, 27-51.

Brothers L, Ring B (1992). A neuroethological framework for the representation of minds. Journal of Cognitive Neuroscience 4, 107-118.

Brüne M (2003). Social cognition and behaviour in schizophrenia. In The Social Brain: Evolution and Pathology (ed. M. Brüne, H. Ribbert and W. Schiefenhövel), pp. 277-313. John Wiley \& Sons: Chichester.

Brüne M (2005). Emotion recognition, 'theory of mind', and social behavior in schizophrenia. Psychiatry Research 133, 135-147.

Brüne M, Lissek S, Fuchs N, Witthaus H, Peters S, Nicolas V, Juckel G, Tegenthoff M (2008). An fMRI study of theory of mind in schizophrenic patients with 'passivity' symptoms. Neuropsychologia 46, 1992-2001.

Brunet E, Sarfati Y, Hardy-Bayle M, Decety J (2003). Abnormalities of brain function during a nonverbal theory of mind task in schizophrenia. Neuropsychologia 21, 1574-1582.

Callicott JH, Mattay VS, Verchinski BA, Marenco S, Egan MF, Weinberger D (2003). Complexity of prefrontal cortical dysfunction in schizophrenia: more than up or down. American Journal of Psychiatry 160, 2209-2215.

Castelli F, Happé F, Frith U, Frith C (2000). Movement and mind: a functional imaging study of perception and interpretation of complex intentional movement patterns. NeuroImage 12, 314-325.

Corcoran R, Cahill C, Frith CD (1997). The appreciation of visual jokes in people with schizophrenia: a study of 'mentalizing' ability. Schizophrenia Research 24, 319-327.

Doody GA, Götz M, Johnstone EC, Frith C, Cunningham Owens DG (1998). Theory of mind and psychoses. Psychological Medicine 28, 397-405.

Edwards J, Jackson HJ, Pattison PE (2002). Emotion recognition via facial expression and affective prosody in schizophrenia : a methodological review. Clinical Psychology Review 22, 789-832.

Fahim C, Stip E, Mancini-Marie A, Mensour B, Boulay JC, Leroux J, Beaudoin G, Bourgoin P, Beauregard M (2005). Brain activity during emotional negative pictures in schizophrenia with and without flat affect: an fMRI study. Psychiatry Research: Neuroimaging 140, 1-15.

Fakra E, Salgado-Pineda P, Delaveau P, Hariri AR, Blin O (2008). Neural bases of different cognitive strategies for facial affect processing in schizophrenia. Schizophrenia Research 100, 191-205.

Frith CD (1992). The Cognitive Neuropsychology of Schizophrenia. Lawrence Erlbaum Associates Inc.: Hillsdale, NJ.

Gallwitz A, Lehrl S, Blaha L (1992). Short Test for General Intelligence [in German]. Hogrefe: Göttingen.

Gregory C, Lough S, Stone V, Erzinclioglu S, Martin L, Baron-Cohen S, Hodges JR (2002). Theory of mind in patients with frontal variant frontotemporal dementia and Alzheimer's disease: theoretical and practical implications. Brain 125, 752-764.

Greig TC, Bryson BJ, Bell MD (2004). Theory of mind performance in schizophrenia: diagnostic, symptom, and neuropsychological correlates. Journal of Nervous and Mental Disease 192, 12-18.

Grèzes J, Berthoz S, Passingham RE (2006). Amygdala activation when one is the target of deceit: did he lie to you or to someone else? NeuroImage 30, 601-608.

Gur RE, McGrath C, Chan RM, Schroeder L, Turner T, Turetsky BI, Kohler C, Alsop D, Maldajian J, Ragland JD, Gur RC (2002). An fMRI study of facial emotion processing in patients with schizophrenia. American Journal of Psychiatry 159, 1992-1999.

Hempel A, Hempel E, Schönknecht P, Stippich C, Schröder J (2003). Impairment in basal limbic function in schizophrenia during affect recognition. Psychiatry Research: Neuroimaging 122, 115-124.

Herold R, Tényi T, Lénárd K, Trixler M (2002). Theory of mind deficit in people with schizophrenia during remission. Psychological Medicine 32, 1125-1129.

Holt DJ, Kunkel L, Weiss AP, Goff DC, Wright CI, Shin LM, Rauch SL, Hootnick J, Heckers S (2006). 
Increased medial temporal lobe activation during the passive viewing of emotional and neutral facial expressions in schizophrenia. Schizophrenia Research 82, 153-162.

Horan WP, Nuechterlein KH, Wynn JK, Lee J, Castelli F, Green MF (2009). Disturbances in the spontaneous attribution of social meaning in schizophrenia. Psychological Medicine 39, 635-643.

Inoue $Y$, Yamada $K$, Hirano $M$, Shinohara $M$, Tamaoki $T$, Iguchi H, Tonooka Y, Kanba S (2006). Impairment of theory of mind in patients in remission following first episode of schizophrenia. European Archives of Psychiatry and Clinical Neuroscience 256, 326-328.

Janssen I, Krabbendam L, Jolles J, van Os J (2003). Alterations in theory of mind in patients with schizophrenia and non-psychotic relatives. Acta Psychiatrica Scandinavica 108, 110-117.

Johnston PJ, Devir H, Karayanidis F (2006). Facial emotion processing in schizophrenia: no evidence for a deficit specific to negative emotions in a differential deficit design. Psychiatry Research 143, 51-61.

Kelemen O, Kéri S, Must A, Benedek G, Janka Z (2005). No evidence for impaired 'theory of mind' in unaffected first-degree relatives of schizophrenia patients. Acta Psychiatrica Scandinavica 110, 146-149.

Kern RS, Green MF, Fiske AP, Kee KS, Lee J, Sergi MJ, Horan WP, Subotnik KL, Sugar CA, Nuechterlein KH (2009). Theory of mind deficits for processing counterfactual information in persons with chronic schizophrenia. Psychological Medicine 39, 645-654.

Kington JM, Jones LA, Watt AA, Hopkins EJ, Williams J (2000). Impaired eye expression recognition in schizophrenia. Journal of Psychiatric Research 34, 341-347.

Kosaka K, Omori M, Murata T, Iidaka T, Yamada H, Okada T, Takahashi T, Sadato N, Itoh H, Yonekura Y, Wada Y (2002). Differential amygdala response during facial recognition in patients with schizophrenia: an fMRI study. Schizophrenia Research 57, 87-95.

LeDoux J (2007). The amygdala. Current Biology 17, R868-R874.

Lehrl S (1977). Multiple-choice Vocabulary Test [in German]. Straube: Erlangen.

Li H, Chan RCK, McAlonan GM, Gong Q (2009). Facial emotion processing in schizophrenia: a meta-analysis of functional neuroimaging data. Schizophrenia Bulletin. Published online: 30 March 2009. doi:10.1093/schbul/ sbn190.

Liberzon I, Phan KL, Decker LR, Taylor SF (2003). Extended amygdala and emotional salience: a PET activation study of positive and negative affect. Neuropsychopharmacology 28, 726-733.

López-Ibor JJ, López-Ibor M, Méndez M, Morón M, Ortiz-Terán L, Fernandez A, Diaz-Marsá M, Ortiz T (2008). The perception of emotion free faces in schizophrenia: a magneto-encephalography study. Schizophrenia Research 98, 278-286.

Loughland CM, Williams LM, Gordon E (2002). Visual scanpaths to positive and negative facial emotions in an outpatient schizophrenia sample. Schizophrenia Research 55, 159-170.
Mandal MK, Pandey R, Prasad AB (1998). Facial expression of emotions and schizophrenia: a review. Schizophrenia Bulletin 24, 399-412.

Manor BR, Gordon E, Williams LM, Rennie CJ, Bahramali H, Latimer CR, Barry RJ, Meares RA (1999). Eye movements reflect impaired face processing in patients with schizophrenia. Biological Psychiatry 46, 963-969.

Margraf J (1994). Short Diagnostic Structured Interview for Mental Disorders : Mini-DIPS [in German]. Springer: Berlin.

Mizrah R, Bagby RM, Zipursky RB, Kapur S (2005). How antipsychotics work: the patients' perspective. Progress in Neuro-Psychopharmacology and Biological Psychiatry 29, 859-864.

Mo S, Su Y, Chan RCK, Liu J (2008). Comprehension of metaphor and irony in schizophrenia during remission: the role of theory of mind and IQ. Psychiatry Research 157, 21-29.

Pelphrey KA, Morris JP, McCarty G (2004). Grasping the intentions of others: the perceived intentionality of an action influences activity in the superior temporal sulcus during social perception. Journal of Cognitive Neuroscience 16, 1706-1716.

Penn, DL, Combs DR, Ritchie M, Franzis J, Cassisi J, Morris S, Townsend M (2000). Emotion recognition in schizophrenia: further investigation of generalized versus specific deficit models. Journal of Abnormal Psychology 109, 512-516.

Penn DL, Sanna LJ, Roberts DL (2008). Social cognition in schizophrenia: an overview. Schizophrenia Bulletin 34, 408-411.

Pinkham AE, Gur RE, Gur RC (2007). Affect recognition deficits in schizophrenia: neural substrates and psychopharmacological implications. Expert Review of Neurotherapeutics 7, 807-816.

Pinkham AE, Hopfinger JB, Pelphrey KA, Piven J, Penn DL (2008). Neural bases for impaired theory of mind in schizophrenia and autism spectrum disorder. Schizophrenia Research 99, 164-175.

Pinkham AE, Penn DL, Perkins DO, Lieberman J (2003). Implications for the neural basis of social cognition for the study of schizophrenia. American Journal of Psychiatry 160, 815-824.

Premack D, Woodruff G (1978). Does the chimpanzee have a theory of mind? Behavioral and Brain Sciences 4, 515-526.

Russell TA, Rubia KR, Bullmore ET, Soni W, Suckling J, Brammer MJ, Simmons A, Williams SCR, Sharma T (2000). Exploring the social brain in schizophrenia. Left prefrontal underactivation during mental state attribution. American Journal of Psychiatry 157, 2040-2042.

Saxe R, Xiao D-K, Kovacs G, Perrett DI, Kahnwisher N (2004). A region of right posterior superior temporal sulcus responds to observed intentional actions. Neuropsychologia 42, 1435-1446.

Seiferth NY, Pauly K, Habel U, Kellermann T, Shah NJ, Ruhrmann S, Klosterkötter J, Schneider F, Kircher T (2008). Increased neural response related to neutral faces in individuals at risk for psychosis. NeuroImage 40, 289-297.

Shamay-Tsoory SG, Shur S, Barcai-Goodman L, Medlovich S, Harari H, Levkovitz Y (2007). Dissociation 
of cognitive from affective components of theory of mind in schizophrenia. Psychiatry Research 149, 11-23.

Shamay-Tsoory SG, Tomer R, Aharon-Peretz J (2005).

The neuroanatomical basis of understanding sarcasm and its relationship to social cognition. Neuropsychology 19, 288-300.

Surguladze S, Russell T, Kucharska-Pietura K, Travis MJ, Giampietro V, David AS, Phillips ML (2006). A reversal of the normal pattern of parahippocampal response to neutral and fearful faces is associated with reality distortion in schizophrenia. Biological Psychiatry 60, 423-421.
Tzourio-Mazoyer N, Landeau B, Papathanassiou D, Crivello F, Etard O, Delcroix N, Mazoyer B, Joliot M (2002). Automated anatomical labeling of activations in SPM using a macroscopic anatomical parcellation of the MNI MRI single-subject brain. NeuroImage 15, 273-289.

Walter B, Blecker C, Kirsch P, Sammer G, Schienle A, Stark R, Vaitl D (2003). MARINA: an easy to use tool for the creation of MAsks for Region of INterest Analyses [Abstract]. Presented at the 9th International Conference on Functional Mapping of the Human Brain, 19-22 June 2003, New York, NY. (Available on CD-Rom in NeuroImage vol. 19, no. 2.) 